\title{
Genipin Cross-Linked Polyvinyl Alcohol-Gelatin Hydrogel for Bone Regeneration
}

\author{
Thi-Hiep Nguyen', Reiza Ventura1, Young-Ki Min², Byong-Taek Lee ${ }^{1,3}$ \\ ${ }^{1}$ Department of Regenerative Medicine, College of Medicine, Soonchunhyang University, Asan, South Korea \\ ${ }^{2}$ Department of Physiology, College of Medicine, Soonchunhyang University, Asan, South Korea \\ ${ }^{3}$ Institute of Tissue Regeneration, College of Medicine, Soonchunhyang University, Asan, South Korea \\ Email: Ibt@sch.ac.kr
}

Received 13 June 2016; accepted 29 July 2016; published 2 August 2016

Copyright (C) 2016 by authors and Scientific Research Publishing Inc.

This work is licensed under the Creative Commons Attribution International License (CC BY).

http://creativecommons.org/licenses/by/4.0/

(c) (i) Open Access

\begin{abstract}
Polyvinyl alcohol gelatin hydrogels were fabricated using genipin as a crosslinking agent for bone regeneration application. Optimized formulation of PVA-GE hydrogel was fabricated using genipin as crosslinking agent. Characterizations such as FTIR, morphology, porosity, pore size, degradation and swelling rate were investigated. Bone regeneration potential of optimized genipin cross-linked polyvinyl alcohol-gelatin (PVA20) hydrogels was assessed by implanting in rabbit's femur defect for 1,5 and 15 weeks period. Results showed interconnected porosity as observed in scanning electron microscopy and successful crosslinking as confirmed by FTIR analysis. Increased porosity $(92 \% \pm 2.46 \%)$ and pore size distribution $(100-200 \mu \mathrm{m})$ were also observed as well as decrease in swelling rate $(426 \% \pm 10.50 \%)$. Bone formation was evident in micro-CT after 5 and 15 days of in vivo implantation period. Micro-CT analysis showed $32.67 \%$ increased bone formation of PVA-GE hydrogel defect compared with negative control after 15 weeks of in-vivo implantation. Histological analyses showed no inflammatory reaction post implantation and increase in cell matrix formation after 5 and 15 weeks. The combined physical and chemical method of crosslinking promises improved mechanical properties of PVA-GE hydrogel making it a potential scaffold for bone tissue engineering applications.
\end{abstract}

\section{Keywords}

PVA-Gelatin, PVA, Gelatin, Genipin, Hydrogel, Bone Regeneration

\section{Introduction}

Hydrogels based on both natural and synthetic polymers have continued to be of interest for the new field of 
tissue engineering for repairing and regenerating a wide variety of tissues and organs [1]. It has excellent biocompatibility, easily controlled degradability and vascularization inductivity [2]. Moreover, hydrogel systems can be easily formed into different shapes and can be filled into bone defects during implantation [3]. It provides a highly swollen three-dimensional (3D) environment similar to soft tissues and allows diffusion of nutrients and cellular waste through its elastic networks, which is essential in bone and tissue regeneration. However, hydrogels derived from natural polymers that use in hard tissue regeneration have fast degradation rate despite having desirable properties [4]. On the other hand, synthetic hydrogels can be prepared with tailored and highly reproducible chemical characteristics, thereby enabling the required degradation properties [5]. Recent strategies have focused on composite hydrogels by combining different degradable or non-degradable polymers with tailored chemistries in order to create bioactive systems with customized functional properties [6] [7]. Studies with several hydrogel systems consisting of either natural polymers such as gelatin, hyaluronic acid and chitosan, or synthetic polymers such as polyvinyl alcohol or polyethylene glycol have been developed for bone regeneration [8]-[12].

Biomimetic hydrogel scaffolds consisting of natural and synthetic constituents offer both biocompatibility and structural versatility [13]-[15]. PVA hydrogel is known for high degree of swelling in water, inherent low toxicity, good biocompatibility and desirable physical properties. It has been used clinically in animals for articular cartilage healing [16]. However, PVA hydrogel has limitations in cell-biomaterials interaction and should be combined with other tissues inducing materials to accelerate the healing process [17]. Gelatin on the other hand, is non-toxic, biodegradable and water soluble protein derived from collagen [18]. Gelatin is a natural polymer derived from the hydrolysis of collagen. It is biodegradable, biocompatible and low in cost [19]. It has lower immunogenicity, good solubility in aqueous systems and a sol-gel transition at $30^{\circ} \mathrm{C}$ [20]. Moreover, it has excellent biocompatibility, promotes good cell adhesion, proliferation and non-carcinogenic [21] [22]. In addition, gelatin can be cross-linked or modified with the inclusion of other materials to significantly alter its mechanical and biochemical properties. Composite of PVA and gelatin for bone regeneration has been investigated using different methods such as electrospinning, hydrogel and salt leaching to assess its suitability for bone regeneration PVA is used as a biomaterial due to its biocompatibility, nontoxicity, non-carcinogenicity and excellent swelling properties. PVA needs to be cross-linked prior to the fabrication of hydrogel due to its water solubility [23]. Crosslinking methods of PVA-GE hydrogel fabrication have been reported by different studies such as repeated freeze-thaw cycles, changing the degree of hydrolysis of the PVA, adding a foaming agent [24]-[29].

Genipin was utilized as a crosslinking agent in PVA-GE hydrogel fabrication. It is a naturally occurring cross-linking agent, which is isolated from the fruits of the gardenia jasmin-oides plant [13]. It reacts with primary amine groups to fix biological tissues and is significantly less cytotoxic than other chemical cross-linkers, such as glutaraldehyde, diisocyanates and epoxides [14]. It was used as a crosslinking agent due to its ability to improve mechanical properties and has non-toxic properties. PVA-GE hydrogel has been used in different studies for vascular tissue engineering, wound healing, food engineering, drug delivery applications, cell encapsulations and 3-D scaffolds for tissue engineering and biomedical application [30]-[38]. The objective of this study is to provide a simple, easy and effective hydrogel fabrication method for bone tissue regeneration and to assess the effect of genipin on the physical and chemical composition of the fabricated hydrogels and its bone regeneration potential. Physical properties were investigated using SEM, porosity, pore size distribution, water uptake and FTIR before and after crosslinking. Bone regeneration potential of the hydrogels was observed by implanting cross-linked PVA-GE hydrogels in a rabbit femur defect for 5 and 15 weeks.

\section{Experimental Procedure}

\subsection{Hydrogel Preparation}

PVA and GE solution were prepared separately. Solution containing varying amount of PVA (wt/v) PVA (Sigma, USA) and varying amount of gelatin (wt/v) (approx. 300 Bloom, Sigma, USA) was dissolved in deionized water at $80^{\circ} \mathrm{C}$ and mixed for 2 hours. Genipin (Wako, Korea) solution was prepared by dissolving $0.05 \%$ w/v of genipin powder in PBS solution and mixed for 30 minutes. PVA and GE solution were mixed together with varying volume of $0.05 \%$ genipin solution of $0 \mathrm{ml}, 40 \mathrm{ml}, 60 \mathrm{ml}$ and $80 \mathrm{ml}$ (Table 1 ) at room temperature until a homogenous mixture was obtained. The mixture was poured into a polyvinyl chloride (PVC) molds and allowed to undergo crosslinking reaction for 2 days at room temperature. Table 1 shows different concentration of PVA and gelatin together with different volume of $0.05 \%$ genipin solution used in fabrication of PVA-gelatin hydrogel. 
Table 1. Different combination of polyvinyl alcohol and gelatin with varying volume of $0.05 \%$ genipin solution used for PVA-GE hydrogel fabrication.

\begin{tabular}{ccc}
\hline Samples & PVA:Gelatin ratio & Genipin volume (0.05\%) \\
\hline PVA10 & $10: 10$ & 80 \\
PVA20 & $20: 20$ & 60 \\
PVA30 & $30: 30$ & 40 \\
PVA50 & $50: 50$ & 0 \\
\hline
\end{tabular}

\subsection{Characterization of PVA-GE Hydrogel}

Fabricated PVA-GE hydrogels with different composition were first determined its morphology, compressive strength, swelling rate and degradation.

Scanning Electron Microscopy. Hydrogel morphology before and after crosslinking were evaluated under scanning electron microscopy (JEOL, Japan) at an acceleration voltage of $25 \mathrm{kV}$. Samples were prepared prior to SEM observation. PVA-GE hydrogels were fabricated as stated above, dehydrated in ethanol series and hexamethyldisilazane (HMDS, Sigma, USA) was added drop wise for critical point drying. Dried PVA-GE hydrogels were mounted in sample holder and sputter coated (JFC-1200, JEOL, Japan) with thin platinum layer and viewed under scanning electron microscope.

Compressive strength. Compressive strength of hydrogels was measured to determine the mechanical strength of the hydrogels. PVA-GE hydrogels were wetted with the use of phosphate buffer saline (PBS) to simulate real conditions. The hydrogel solution was cast into a $5 \mathrm{~mm}$ petri dish and wet hydrogel samples were cut into square shapes (5 mm in length, $5 \mathrm{~mm}$ width and $5 \mathrm{~mm}$ thickness). A uniaxial displacement was applied to the specimen at a crosshead speed of $0.5 \mathrm{~mm} / \mathrm{min}$ and the load was measured with a $20-\mathrm{g}$ load cell. The reported results were the averages of five trials of the samples, along with the corresponding standard deviations.

Swelling rate. The weights of the completely dried hydrogels were determined using an analytical balance. Hydrogels (square shape with $1 \mathrm{~mm}$ thickness and $1 \mathrm{~mm}$ width) were immersed in PBS at $37^{\circ} \mathrm{C}$ in an incubator for 24 hours. The swollen hydrogels were gently blotted with filter paper to remove excess surface water and final weight of the hydrogels were recorded. The swelling rate (\%) of the hydrogels was calculated as:

$$
\frac{W_{s}-W_{o}}{W_{o}} * 100
$$

where $W_{s}$ is the weight of swelling hydrogel and $W_{o}$ is the initial weight of dry hydrogel.

Degradation rate. Degradation rate of hydrogels were measured with the use of lysozyme solution for 60 days. Initial weight of the hydrogels were recorded $\left(W_{i}\right)$ and incubated in PBS solution with $10 \mathrm{mg} / \mathrm{ml}$ lysozyme at $37^{\circ} \mathrm{C}$. After 60 days final weight of PVA-GE hydrogels with different combinations of PVA and gelatin were obtained and recorded, hydrogels were blotted dry using a filter paper and final weight were obtained $\left(W_{f}\right)$. Lysozyme solution was changed every two days. Degradation rate (\%) was calculated as: Degradation rate (\%):

$$
\frac{W_{i}-W_{f}}{W_{i}} * 100
$$

Optimized samples will be chosen based on compressive strength, degradation rate and swelling rate.

FTIR analysis. Cross-linked PVA20 hydrogel and Uncross-linked PVA50 hydrogel which served as a control were characterized by attenuated reflectance Fourier transform spectroscopy (PerkinElmer, USA). The infrared spectra of the samples were measured over a wavelength range of $2000-800 \mathrm{~cm}^{-1}$. All spectra in the spectral range were collected by 64 scans with a resolution of $4 \mathrm{~cm}$.

Porosimetry. To analyze the porosity and pore size, PVA20 and PVA50 hydrogels $(5 \mathrm{~mm}$ in height, $5 \mathrm{~mm}$ in width and $10 \mathrm{~mm}$ in thickness) were evaluated using Mercury porosimeter (Quantachrome Instruments, USA) and analyzed using Poremaster software.

\subsection{In-Vivo Evaluation of Cross-Linked PVA-GE Hydrogel}

Implantation of PVA-GE (PVA20) hydrogel in rabbit's femur defect. All in-vivo procedures were carried out according to the protocol approved by the Animal Ethical Committee of Soonchunhyang University for the 
care and use of laboratory animals. Bone regeneration potential of PVA-GE (PVA20) hydrogels were assessed by implanting PVA-GE in rabbits' femur. Two different groups were used in the in-vivo study; the negative control which consisted of defect only and the PVA-GE group wherein the defect were implanted with PVA20 hydrogel for 1, 5 and 15 weeks. New Zealand white rabbit $(n=18)$, were kept in light and temperature controlled environment and were given food and water. Rabbits were sanitized using $75 \%$ alcohol and cleaned with iodine, intramuscular injection of $0.8 \mathrm{ml}$ of Zoletil 50 was injected prior to operation. A longitudinal incision was done on the parietal part of the femur, a $3 \mathrm{~mm}$ defect was made using a trephine drill. Prior to operation, PVA-GE hydrogels were sterilized by washing with PBS and exposed to UV light for 15 minutes. Sterilized PVA-GE (3 $\mathrm{mm}$ by $5 \mathrm{~mm}$ ) hydrogel was inserted into the defect or left empty as a control. Laboratory animals were kept and fed ad libitum. The rabbits were sacrificed 1, 5 and 15 weeks after implantation, and the entire portion of the femur defect was removed. The samples were immersed in a $10 \%$ buffered formaldehyde solution at room temperature to preserve the tissues until further use.

Micro-CT analysis. Micro CT was utilized to determine the new bone formation in the femoral defect site after 1, 5 and 15 weeks. Extracted femurs were fixed with formalin overnight and covered with paraffin. Prepared samples were scanned using micro-CT to obtain micro CT scans over the region of interest (ROI) of the samples. Micro Ct scans were then converted to 3D using a CT Analyzer software (CTAn) and viewed using a 3-D Visualization software (CTVol). 3D data were then reconstructed to obtain quantitatively the bone volume $(B V)$ and tissue volume (TV) [39] [40]. Percent bone volume was calculated to measure the new bone volume using the formula below:

$$
\text { Bone Volume }(\%)=\frac{B V}{T V} * 100
$$

Histological staining analysis. Harvested rabbit femurs were fixed in $10 \%$ formalin solution for 2 days and decalcified using 5\% nitric acid for 3 days at room temperature. Decalcified samples were fixed in paraffin and cut using HM 325 microtome (Thermo Scientific, USA). Tissue section with $5 \mu \mathrm{m}$ thin were fixed in glass slides, deparaffinized in xylene series and dried in alcohol series. Tissue slides were stained with Hematoxylin and eosin (H\&E) and Masson's Trichrome stain and viewed under bright field microscope (Olympus BX53 system microscope (Olympus, Japan) equipped with CellSens software to assessed detailed new bone formation and collagen formation.

Stastistical analysis. The values are expressed as mean \pm standard deviation in each experiment. The results were analyzed statistically using $n=3$ one-way ANOVA with post-hoc correction (Bonferroni method). All analyses were carried out using GraphPad Prism 5 with a confidence level of $p<0.05$ to determine the statistical significance of data obtained in the experiments.

\section{Results}

SEM, compressive strength, degradation and swelling rate. Hydrogels with different PVA and gelatin ratio were first evaluated for its morphology with the use of SEM (Figure 1). SEM micrographs showed that PVA10 hydrogels containing 10:10 PVA-GE ratio has porous morphology with interconnected porosity and presence of open and closed pores (Figure 1(A)). In PVA20, enhanced interconnected porosity was observed (Figure 1(B)) while in PVA30 decreased in pore interconnection was noted due to increase of close pores (Figure 1(C)). However PVA50 has dense morphology with no porosity interconnection was noted (Figure 1(D)). In Figure 2 showed results of compressive strength, degradation rate and swelling rate of different formulation of PVA-GE hydrogels. PVA10 has a compressive strength of $0.3 \mathrm{MPa}$, a degradation rate of $56.77 \%$ and a swelling rate of 100\%. PVA20 hydrogels containing 20:20 PVA-GE ratio has a compressive strength of $7.5 \mathrm{MPa}$, highest and significantly different among the samples, a degradation rate of $57.81 \%$ and has a lowest swelling rate of $70 \%$. PVA30 hydrogels that contained 30:30 PVA-GE ratio also has a compressive strength of $1.2 \mathrm{MPa}$, the lowest value among the samples, degradation of $48.89 \%$ and an increased swelling rate of $150 \%$ compared with PVA10 and PVA20 hydrogels. PVA50 hydrogels which was uncross-linked and used as a control, has very poor mechanical strength and degradation rate, degraded immediately upon contact with water and has a $200 \%$ swelling rate.

Based on the compressive strength, degradation and swelling rate, PVA20 hydrogels containing 20:20 PVA-GE ratio and with $60 \mathrm{ml}$ of $0.05 \%$ genipin solution were chosen as the optimized sample. It has the highest compressive strength value (7.5 MPa) and the lowest value of swelling rate (70\%) among the PVA-GE hydrogels. Optimized hydrogels (PVA20) were further characterized using FTIR analysis and porosimetry. 
FTIR analysis. FTIR analysis was carried out determine the structural modification of PVA-GE hydrogel before and after crosslinking. The FTIR spectra of uncross-linked hydrogel, PVA50 (A) and cross-linked hydrogel, PVA20 (B) are shown in Figure 3. The peak at $1640 \mathrm{~cm}^{-1}$ corresponds to $\mathrm{C}=\mathrm{O}$ stretching that indicated amide I and the peak between $1550 \mathrm{~cm}$ and $1500 \mathrm{~cm}^{-1}$ corresponds to -NH bending that corresponded to amide II. The peaks at $1088 \mathrm{~cm}^{-1}$ and $1277 \mathrm{~cm}^{-1}$ indicated the C-O stretching of secondary alcoholic and ester group which indicated amide III. Presence of peaks from $900-100 \mathrm{~cm}^{-1}$ were observed in cross-linked PVA-GE hydrogel indicated formation of intra and intermolecular crosslinking bonds by the formation of heterocyclic structure of genipin with primary amine groups. Increased in peaks were observed in the cross-linked PVA-GE hydrogel compared with uncross-linked PVA-GE hydrogels indicated successful crosslinking between PVA and gelatin using genipin.

Porosimetry. Pore size distribution histogram (Figure 4) revealed smaller pore size distribution in PVA50 (5 - 20 um) as seen in Figure 4(C) compared with PVA20 which has bigger (100 - 200 um) pore size distribution as seen in Figure 4(D). Porosity of PVA50 were also evaluated (Figure 4(E)) and increased porosity with 92\% $\pm 2.46 \%$ was observed in PVA20 hydrogel compared with PVA50 which has only $71 \% \pm 3.12 \%$ porosity.
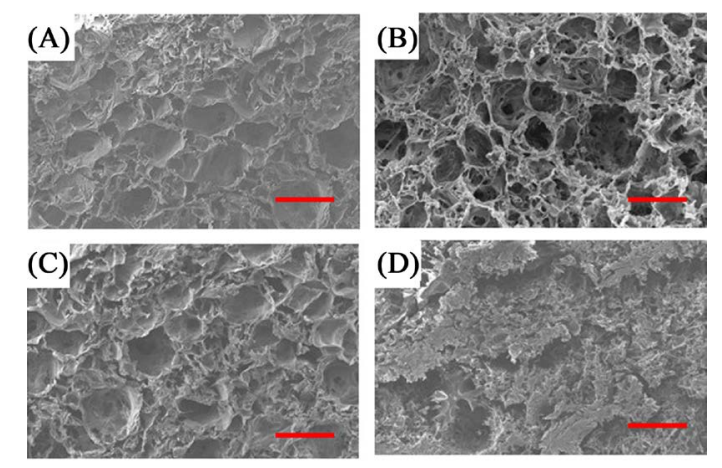

Figure 1. Scanning electron micrographs of PVA-GE with different PVA and gelatin composition, PVA10 (A), PVA20 (B), PVA30 (C) and PVA50 (D). Scale bar indicates $100 \mu \mathrm{m}$.
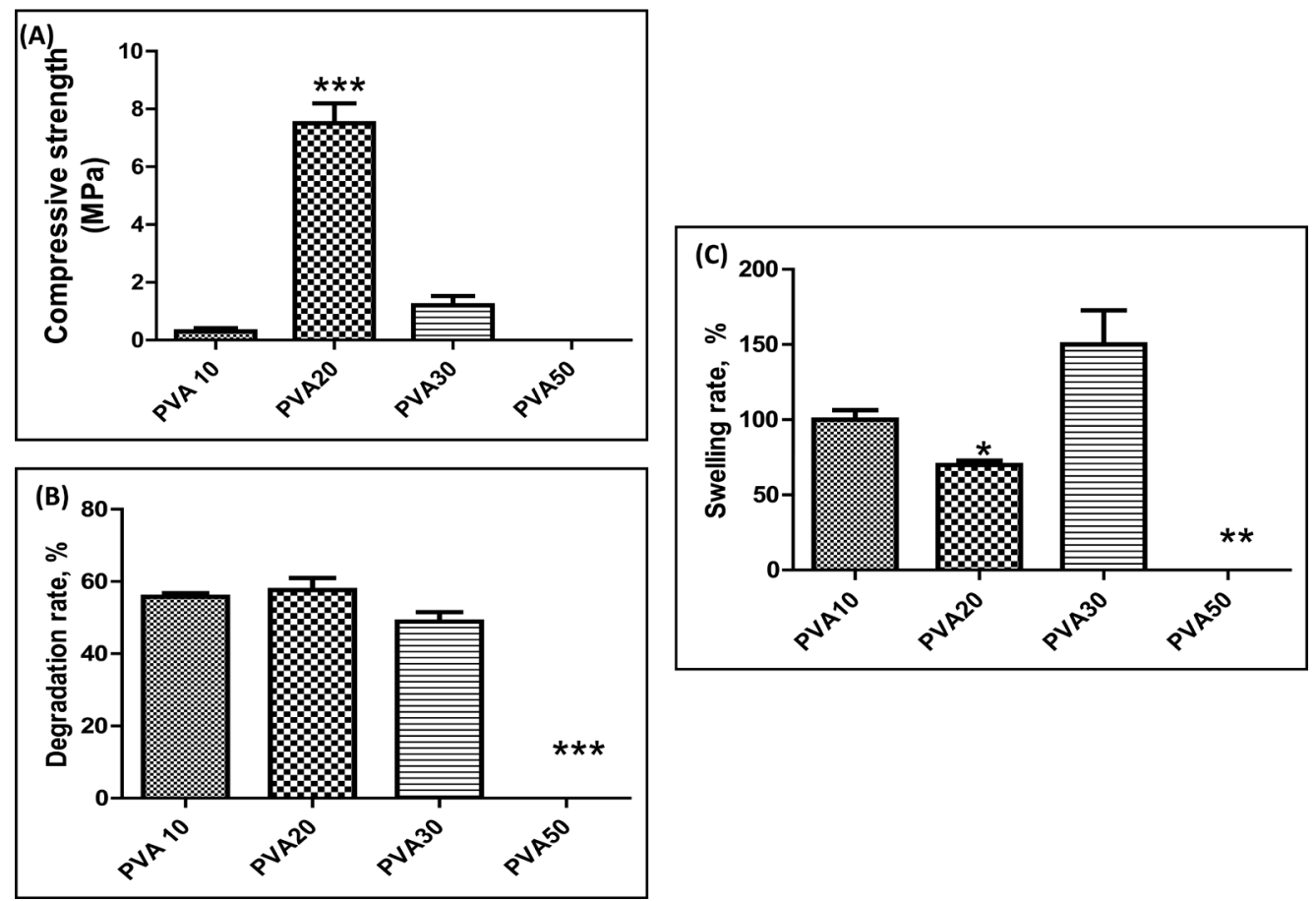

Figure 2. Characterization of PVA-GE hydrogels with different PVA and gelatin composition. Compressive strength (A), degradation rate (B) and swelling rate (C) of hydrogels with different PVA-Gelatin ratio. 


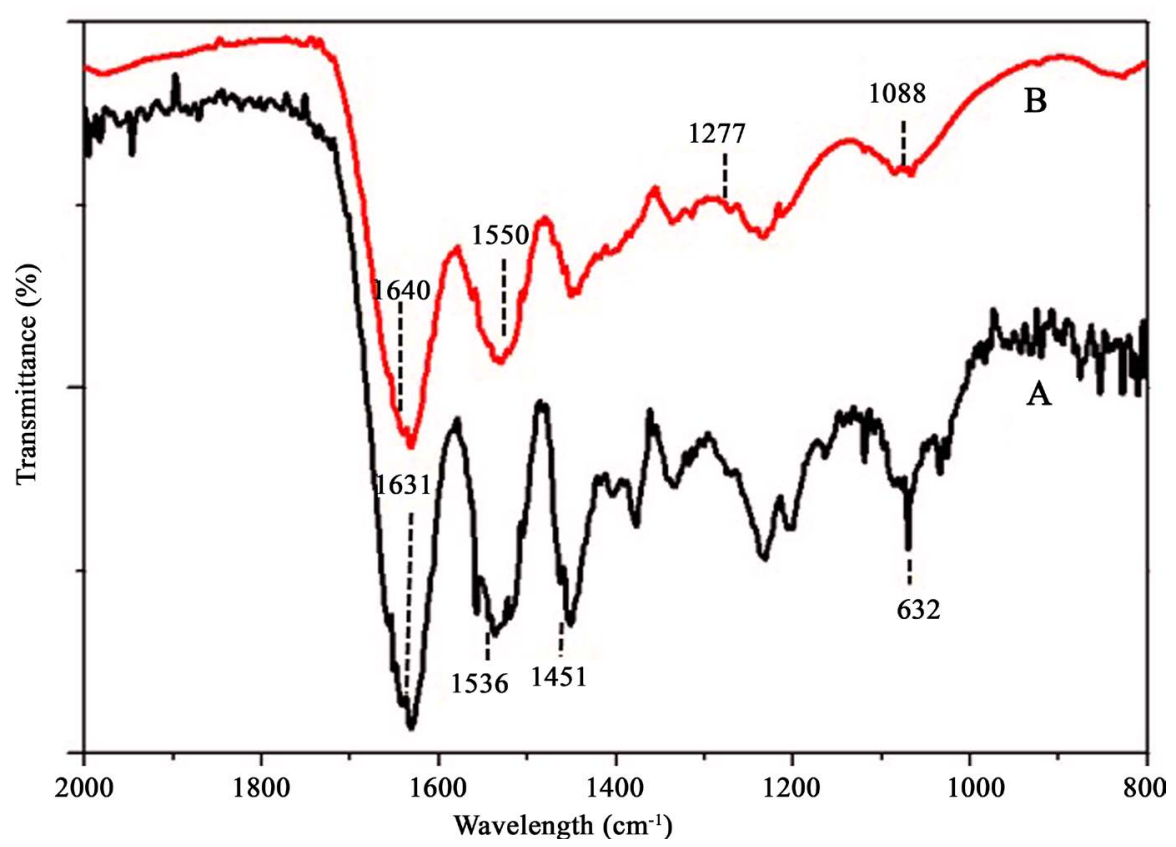

Figure 3. FT-IR spectrum of uncrosslinked (A) and cross-linked (B) PVA-GE hydrogels.
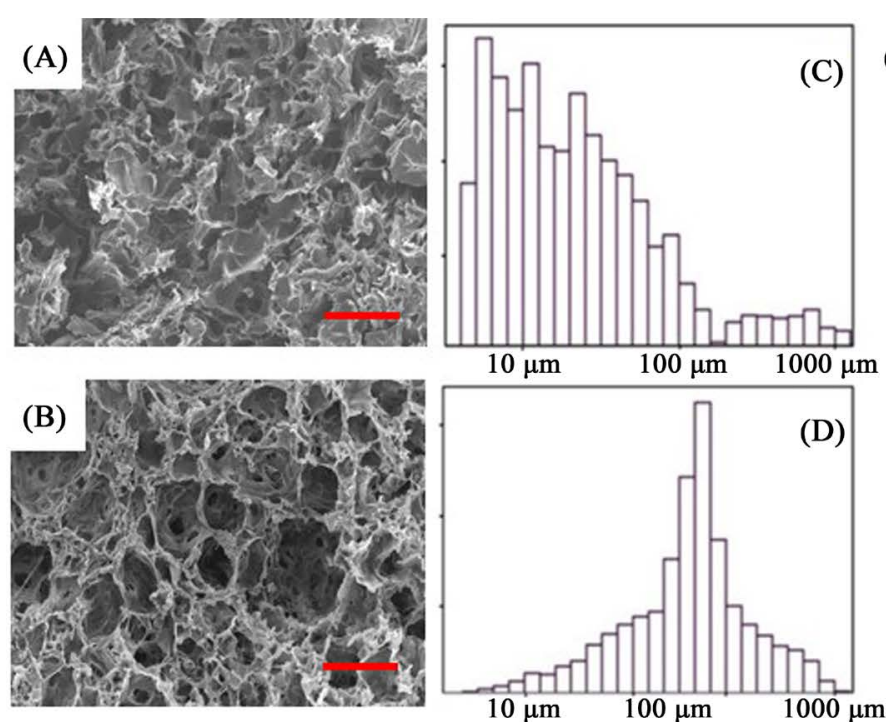

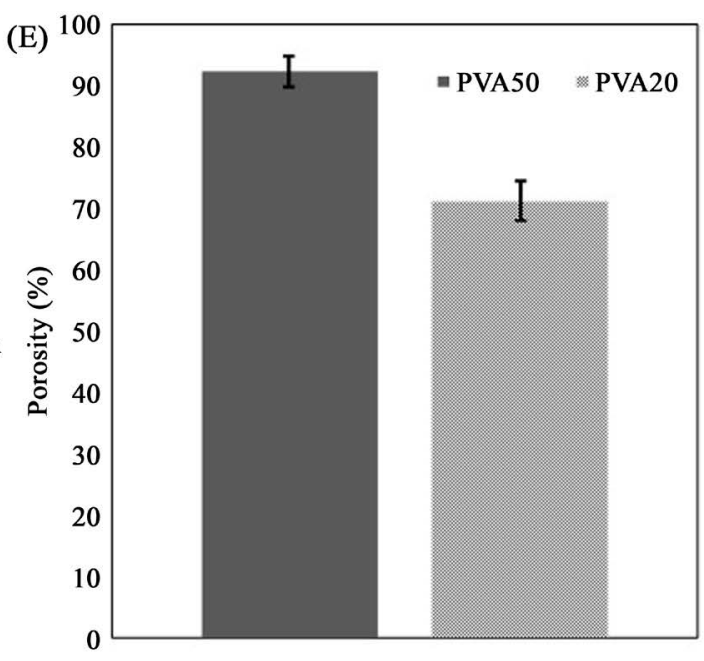

Figure 4. Morphology and corresponding pore size distribution of PVA50 ((A) and (C)) PVA20 hydrogels ((B) and (D)) and $\%$ Porosity (E). Scale bar indicates $100 \mu \mathrm{m}$.

\section{In Vivo Evaluation of PVA-GE Hydrogel}

PVA20 was implanted in rabbit's femur to determine its bone regeneration potential. Animals were euthanized and samples were extracted after 1, 5 and 15 weeks for Micro-CT and histological staining analyses.

Micro-CT analysis. Micro-CT images of negative control and PVA20 hydrogel after 1, 5 and 15 weeks of implantation were showed in Figure 5. After 1 week of in-vivo implantation, bone formation on the interface of the defect site were observed both in the negative control (Figure 5(A1)) and PVA-GE defect (Figure 5(A2) \& Figure 5(A3)). Increased bone formation was observed after 5 weeks in PVA20 hydrogel (Figure 5(B2) \& Figure 5(B3)) defect compared with negative control (Figure 5(B2)). Bone deposition was visible from the periphery towards the center of the PVA20 hydrogel defect compared with negative control which is visible only on the edges of the defect site (Figure 5(B2) \& Figure 5(B3)). At 15 weeks, bone formation in PVA20 hydrogel 
(Figure 5(C2) \& Figure 5(C3)) defect was observed from the edges to the center compared with the negative control where bone formation is only seen at the periphery of the defect and minimal bone formation at the center (Figure 5(C1)).

Bone volume, \% (Figure 5(D)) was used to quantify the new bone formation in the femoral defect sites using the obtained bone volume (BV) and tissue volume (TV) from micro-CT analysis. Bone volume, \% (bone volume (BV)/tissue volume (TV)) (Figure 5(D)) matched the micro-CT images shown in Figures 5(A)-(C). Bone volume over tissue volume (BV/TV) of the extracted samples were quantified and results showed that no increased in BV/TV was observed in PVA-GE defect $(7.51 \% \pm 1.21 \%)$ compared with the negative control (10.47\% $\pm 2.46 \%$ ) after 1 week of in-vivo implantation. However after 5 weeks, $15 \%$ increased BV/TV was observed in PVA20 defect $(25.14 \% \pm 2.43 \%)$ compared with the negative control $(21.71 \% \pm 3.12 \%)$. At 15 weeks, 32.68\% increase in BV/TV was observed in PVA-GE defect (82.46\% $\pm 4.5 \%)$ compared with the negative control $(62.15 \% \pm 4.21 \%)$.

Histological analyses. Furthermore, histological slides were stained with H\&E and Masson's Trichrome to determine a more detailed analysis of bone formation of the in vivo samples. Hematoxylin and Eosin stained tissue sections (Figure 6) showed no inflammation was observed in the post implantation area including the in-
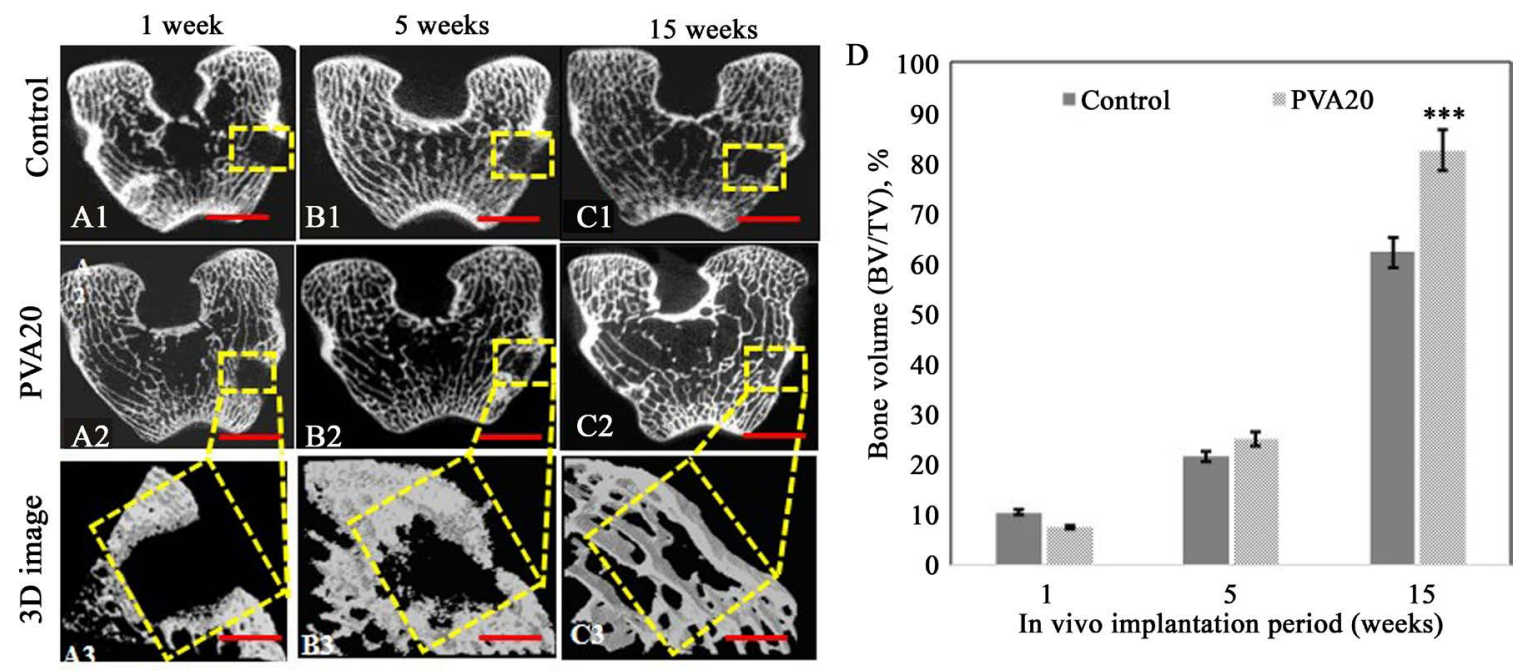

Figure 5. Micro-CT 2D image of negative control ((A1), (B1) and (C1)), PVA-Gehydrogel ((A2), (B2) and (C2)) and 3D images ((A3), (B3) and (C3)) implanted in femur defect for 1, 5 and 15 weeks. Scale bar indicates $1 \mathrm{~mm}$.
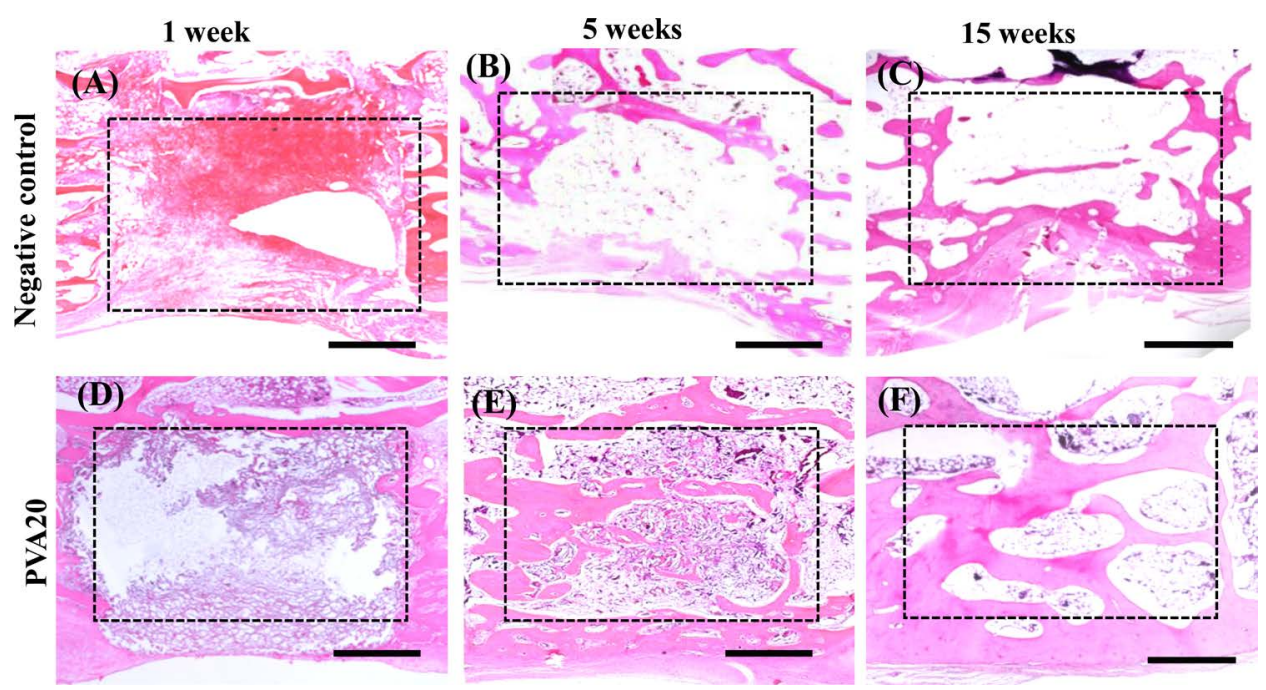

Figure 6. H\&E stained tissue section of positive control (A), negative control ((B)-(D)) and PVA-GE hydrogel ((E)-(G)) after 1,5 and 15 weeks in vivo implantation and positive controls. Scale bar indicates $1 \mathrm{~mm}$. 
terface for both negative and PVA20 defect. At 1 week, dense fibrous connective tissue consisted of fibroblast and blood vessel with an empty space was observed in the defect site of the negative control (Figure 6(A)). However, in PVA20 defect increased in cell matrix formation covering the defect site was observed at the periphery and at the center (Figure 6(D)). At 5 weeks, H\&E stained tissue section of negative control revealed that fibrous tissue composed the majority of the central region with minimal bone formation (Figure 6(B)). In PVA20 defect, new bone formation was observed from the interface of the defect site towards the center with dense matrix formation (Figure 6(E)). At 15 weeks, majority of bone formation in the negative control was observed in the periphery of the defect with fibrous matrix at the center (Figure 6(C)). In PVA20 defect, bone formation was evenly distributed from the interface towards the central region of the defect. (Figure 6(F)). Enhanced bone fixation was also observed in PVA20 defect compared with the negative control.

High magnification of H\&E and Masson's Trichrome stained tissue section micrographs were evaluated for detailed new bone formation (Figure 7). At 5 weeks, H\&E stained tissue sections showed presence of osteoblast and osteocyte surrounding the new bone in the PVA-GE defect site indicating active bone remodeling (Figure 7(A)). At 15 weeks, H\&E stained tissue section showed greater new bone formation in PVA-GE defect with remaining traces of PVA-GE hydrogel (Figure 7(C)). At 5 weeks, high magnification of Masson's Trichrome stained tissue section showed new bone formation was evident on the PVA20 defect site with presence of osteoblasts and osteocytes alongside with collage and cartilage (Figure 7(B)). At 15 weeks, matured cortical bone surrounding with dense collagen and cartilage and traces of PVA20 were still evident in the defect site (Figure 7(D)).

\section{Discussion}

Hydrogels or scaffolds for tissue engineering application need to have a mechanical strength that can withstand handling during operations and in-vivo conditions. Biodegradability is also an important factor to consider during scaffold fabrication. It should be biodegradable over time to allow the cells to produce its own extracellular matrix. Crosslinking of PVA-GE hydrogels using genipin was utilized in this study to be able to produce a scaffold for bone tissue engineering applications. Different PVA and gelatin ratio with different volume of genipin solution with a concentration of $0.05 \%$ was investigated. Based on characterizations, PVA10 which has a minimum amount of PVA to gelation ratio (10:10) with the highest volume of $0.05 \%$ genipin showed the lowest compressive strength among the cross-linked samples. However, PVA30 hydrogels with maximum amount of 30:30 (wt/v) PVA and gelatin and the lowest volume of genipin exhibited low mechanical strength, fastest rate of degradation and highest swelling rate among the cross-linked samples. PVA20 hydrogels were chosen as the optimized samples because of the highest compressive strength, low degradation rate and low swelling rate making it suitable for bone regeneration applications

Overall architecture of scaffolds is another important factor to consider, hydrogels should have a porous structure and interconnected porosity to allow cell penetration and diffusion of nutrients needed by the cells in-

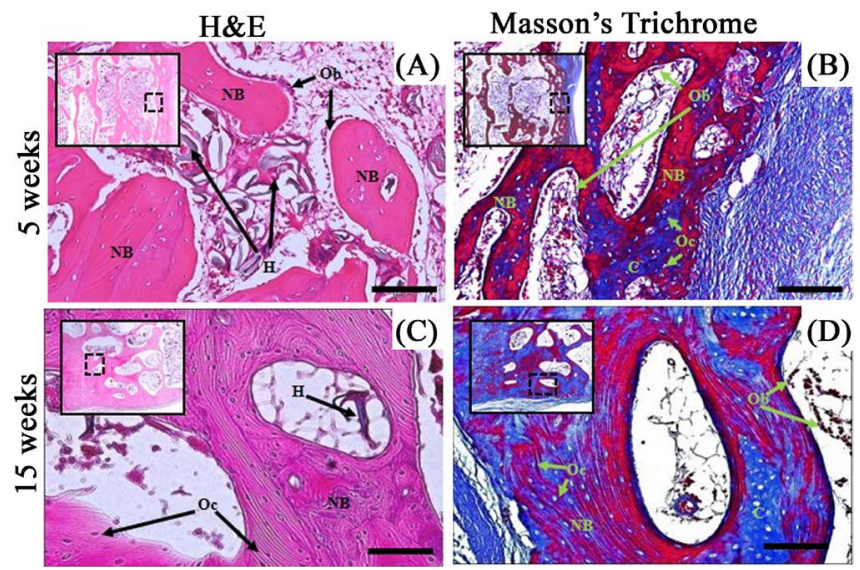

Figure 7. High magnification micrographs of H\&E and Masson's Trichrome stained tissue of PVA-GE hydrogel implant after 5 and 15 weeks in vivo implantation. NB indicates new bone, C for collagen, Ob for osteoblast and Oc for osteocytes. Scale bar indicates $100 \mu \mathrm{m}$. 
side the scaffold and allow waste product diffusion out of the scaffolds. In this study, cross-linked PVA-GE hydrogels has an enhance porosity and pore size compared with uncross-linked PVA-GE hydrogel. Crosslinking of PVA and gelatin using genipin enhanced the mechanical strength, degradability swelling rate as well as porosity and pore size of PVA- GE hydrogels. This is due to the formation of intra and intermolecular crosslinking bonds of heterocyclic structure of genipin with primary amine groups of gelatin and hydrogen bonds between PVA. Increased pore size distribution (100 - $200 \mu \mathrm{m}$ ) was observed (Figure 1) after crosslinking using genipin making it suitable for bone regeneration applications. Porosity, pore interconnectivity and pore size within hydrogels are crucial factors in hydrogel fabrication because it serves as template that facilitates cell attachment, matrix deposition, cell migration, proliferation and signaling and nutrient exchange from the environment to the cell.

PVA20 hydrogels were implanted in rabbit femur to determine the biocompatibility and in vivo bone regeneration capability. Micro-CT and histological analyses revealed increased bone formation in PVA20 defect compared with the control. Increased bone volume (BV/TV) as increased in vivo implantation period was observed in Figure 5. Histological analyses showed no inflammatory reaction in the hydrogel and surrounding areas after 1, 5 and 15 weeks which indicates good biocompatibility. Formation of fibrous tissue was also observed from the periphery of the hydrogel which indicates great affinity of PVA20 with the host tissue. H\&E stained tissue section after 5 weeks implantation showed presence of hydrogel in the defect site and traces of hydrogel were found in 15 week sample which indicated that PVA20 hydrogel degrades slowly over time and can support bone regeneration. These results suggested that PVA20 hydrogel enhanced the osteogenic activity and bone formation in-vivo.

\section{Conclusion}

PVA-GE hydrogel has been successfully fabricated in this study with genipin as a crosslinking agent. Crosslinked PVA-GE hydrogels using genipin improved physical and mechanical properties suitable for tissue engineering applications. In-vivo results showed no inflammatory reaction in the defect site after 1 week of in-vivo implantation, enhanced bone formation and regeneration on rabbit femur defect after 5 and 15 weeks of implantation and could be a potential scaffold for bone regeneration application.

\section{Acknowledgements}

This article was supported by the Basic Science Research Program through the National Research Foundation of Korea (NRF) funded by the Ministry of Education (2015R1A6A1A03032522). The authors would like to thank Mr. Shin-Woo Kim for his assistance of in-vivo operations and Dr. Thi-Hiep Nguyen of Department of Biomedical Engineering, International University, Vietnam National University for her contributions in characterization.

\section{Disclosure Statement}

No competing financial interests exist.

\section{References}

[1] Woerly, S. (1997) Porous Hydrogels for Neural Tissue Engineering. Materials Science Forum, 250, 53-68. http://dx.doi.org/10.4028/www.scientific.net/MSF.250.53

[2] Nguyen, T.P. and Lee, B.T. (2012) Fabrication of Oxidized Alginate-Gelatin-BCP Hydrogels and Evaluation of the Microstructure, Material Properties and Biocompatibility for Bone Tissue Regeneration. Journal of Biomaterials Applications, 27, 311-321. http://dx.doi.org/10.1177/0885328211404265

[3] Gkioni, K., Leeuwenburgh, S.C., Douglas, T.E., Mikos, A.G. and Jansen, J.A. (2010) Mineralization of Hydrogels for Bone Regeneration. Tissue Engineering Part B Reviews, 16, 577-585. http://dx.doi.org/10.1089/ten.teb.2010.0462

[4] Kim, B.S. and Mooney, D.J. (1998) Development of Biocompatible Synthetic Extracellular Matrices for Tissue Engineering. Trends in Biotechnology, 16, 224-230. http://dx.doi.org/10.1016/S0167-7799(98)01191-3

[5] Nair, L.S. and Laurencin, C.T. (2007) Biodegradable Polymers as Biomaterials. Progress in Polymer Science, 32, 762798. http://dx.doi.org/10.1016/j.progpolymsci.2007.05.017

[6] Zustiak, S.P. and Leach, J.B. (2010) Hydrolytically Degradable Poly(Ethylene Glycol) Hydrogel Scaffolds with Tunable Degradation and Mechanical Properties. Biomacromolecules, 11, 1348-1357. http://dx.doi.org/10.1021/bm100137q

[7] Nie, T., Baldwin, A., Yamaguchi, N. and Kiick, K.L. (2007) Production of Heparin-Functionalized Hydrogels for the 
Development of Responsive and Controlled Growth Factor Delivery Systems. Journal of Controlled Release, 122, 287-296. http://dx.doi.org/10.1016/j.jconrel.2007.04.019

[8] Akagawa, Y., Kubo, T., Koretake, K., Hayashi, K., Doi, K., Matsuura, A., et al. (2009) Initial Bone Regeneration around Fenestrated Implants in Beagle Dogs Using Basic Fibroblast Growth Factor-Gelatin Hydrogel Complex with Varying Biodegradation Rates. Journal of Prosthodontic Research, 53, 41-47. http://dx.doi.org/10.1016/j.jpor.2008.08.009

[9] Wang, T.W., Wu, H.C., Huang, Y.C., Sun, J.S. and Lin, F.H. (2006) Biomimetic Bilayered Gelatin-Chondroitin 6 Sulfate-Hyaluronic Acid Biopolymer as a Scaffold for Skin Equivalent Tissue Engineering. Artificial Organs, 30, 141149. http://dx.doi.org/10.1111/j.1525-1594.2006.00200.x

[10] Zhao, F., Yin, Y., Lu, W.W., Leong, J.C., Zhang, W., Zhang, J., et al. (2002) Preparation and Histological Evaluation of Biomimetic Three-Dimensional Hydroxyapatite/Chitosan-Gelatin Network Composite Scaffolds. Biomaterials, 23, 3227-3234. http://dx.doi.org/10.1016/S0142-9612(02)00077-7

[11] Kobayashi, M., Toguchida, J. and Oka, M. (2003) Preliminary Study of Polyvinyl Alcohol-Hydrogel (PVA-H) Artificial Meniscus. Biomaterials, 24, 639-647. http://dx.doi.org/10.1016/S0142-9612(02)00378-2

[12] Park, J.S., Woo, D.G., Sun, B.K., Chung, H.M., Im, S.J., Choi, Y.M., et al. (2007) In Vitro and in Vivo Test of PEG/PCL-Based Hydrogel Scaffold for Cell Delivery Application. Journal of Controlled Release, 124, 51-59. http://dx.doi.org/10.1016/j.jconrel.2007.08.030

[13] Hou, Y., Tsai, S.-Y., Lai, P., Chen, Y. and Chao, P. (2008) Metabolism and Pharmacokinetics of Genipin and Geniposide in Rats. Food and Chemical Toxicology, 46, 2764-2769. http://dx.doi.org/10.1016/j.fct.2008.04.033

[14] Bedran-Russo, A.K.B., Pereira, P.N., Duarte, W.R., Drummond, J.L. and Yamauchi, M. (2007) Application of Crosslinkers to Dentin Collagen Enhances the Ultimate Tensile Strength. Journal of Biomedical Materials Research Part B: Applied Biomaterials, 80, 268-272. http://dx.doi.org/10.1002/jbm.b.30593

[15] Dikovsky, D., Bianco-Peled, H. and Seliktar, D. (2006) The Effect of Structural Alterations of PEG-Fibrinogen Hydrogel Scaffolds on 3-D Cellular Morphology and Cellular Migration. Biomaterials, 27, 1496-1506. http://dx.doi.org/10.1016/j.biomaterials.2005.09.038

[16] Allen, R.A., Seltz, L.M., Jiang, H., Kasick, R.T., Sellaro, T.L., Badylak, S.F., et al. (2010) Adrenal Extracellular Matrix Scaffolds Support Adrenocortical Cell Proliferation and Function in Vitro. Tissue Engineering Part A, 16, 33633374. http://dx.doi.org/10.1089/ten.tea.2010.0005

[17] Badylak, S.F., Freytes, D.O. and Gilbert, T.W. (2009) Extracellular Matrix as a Biological Scaffold Material: Structure and Function. Acta Biomaterialia, 5, 1-13. http://dx.doi.org/10.1016/j.actbio.2008.09.013

[18] Suri, S., Ruan, G., Winter, J. and Schmidth, C. (2013) Microparticles and Nanoparticles. In: Ratner, B., Hoffman, A., Schoen, F. and Lemons, J., Eds., Biomaterials Science: An Introduction to Materials in Medicine, Elsevier, San Diego, 360-388.

[19] Linh, N.T. and Lee, B.T. (2012) Electrospinning of Polyvinyl Alcohol/Gelatin Nanofiber Composites and CrossLinking for Bone Tissue Engineering Application. Journal of Biomaterials Applications, 27, 255-266. http://dx.doi.org/10.1177/0885328211401932

[20] Bohidar, H.B. and Jena, S.S. (1994) Study of Sol-State Properties of Aqueous Gelatin Solutions. The Journal of Chemical Physics, 100, 6888-6895. http://dx.doi.org/10.1063/1.467004

[21] Lee, S.B., Jeon, H.W., Lee, Y.W., Lee, Y.M., Song, K.W., Park, M.H., et al. (2003) Bio-Artificial Skin Composed of Gelatin and $(1 \rightarrow 3),(1 \rightarrow 6)-\beta$-Glucan. Biomaterials, 24, 2503-2511. http://dx.doi.org/10.1016/S0142-9612(03)00003-6

[22] Liang, H.C., Chang, W.H., Lin, K.J. and Sung, H.W. (2003) Genipin-Crosslinked Gelatin Microspheres as a Drug Carrier for Intramuscular Administration: In Vitro And in Vivo Studies. Journal of Biomedical Materials Research Part A, 65, 271-282. http://dx.doi.org/10.1002/jbm.a.10476

[23] Baker, M.I., Walsh, S.P., Schwartz, Z. and Boyan, B.D. (2012) A Review of Polyvinyl Alcohol and Its Uses in Cartilage and Orthopedic Applications. Journal of Biomedical Materials Research Part B: Applied Biomaterials, 100, 14511457. http://dx.doi.org/10.1002/jbm.b.32694

[24] Maria, T.M.C., de Carvalho, R.A., Sobral, P.J.A., Habitante, A.M.B.Q. and Solorza-Feria, J. (2008) The Effect of the Degree of Hydrolysis of the PVA and the Plasticizer Concentration on the Color, Opacity, and Thermal and Mechanical Properties of Films Based on PVA and Gelatin Blends. Journal of Food Engineering, 87, 191-199. http://dx.doi.org/10.1016/j.jfoodeng.2007.11.026

[25] Seo, K.H., Chun, H.J., Lee, W.K. and Nho, Y.C. (2009) In Vitro and in Vivo Biocompatibility of R-Ray Crosslinked Gelatin-Poly (Vinyl Alcohol) Hydrogels. Tissue Engineering and Regenerative Medicine, 6, 414-418.

[26] Liu, Y., Vrana, N., Cahill, P. and McGuinness, G. (2009) Physically Crosslinked Composite Hydrogels of PVA with Natural Macromolecules: Structure, Mechanical Properties, and Endothelial Cell Compatibility. Journal of Biomedical 
Materials Research Part B: Applied Biomaterials, 90, 492-502. http://dx.doi.org/10.1002/jbm.b.31310

[27] Pal, K., Banthia, A.K. and Majumdar, D.K. (2007) Biomedical Evaluation of Polyvinyl Alcohol-Gelatin Esterified Hydrogel for Wound Dressing. Journal of Materials Science: Materials in Medicine, 18, 1889-1894. http://dx.doi.org/10.1007/s10856-007-3061-2

[28] Pawde, S.M. and Deshmukh, K. (2008) Characterization of Polyvinyl Alcohol/Gelatin Blend Hydrogel Films for Biomedical Applications. Journal of Applied Polymer Science, 109, 3431-3437. http://dx.doi.org/10.1002/app.28454

[29] Pal, K., Banthia, A.K. and Majumdar, D.K. (2007) Preparation and Characterization of Polyvinyl Alcohol-Gelatin Hydrogel Membranes for Biomedical Applications. AAPS Pharm SciTech, 8, 21. http://dx.doi.org/10.1208/pt080121

[30] Vrana, N.E., Cahill, P.A. and McGuinness, G.B. (2010) Endothelialization of PVA/Gelatin Cryogels for Vascular Tissue Engineering: Effect of Disturbed Shear Stress Conditions. Journal of Biomedical Materials Research Part A, 94, 1080-1090. http://dx.doi.org/10.1002/jbm.a.32790

[31] Pal, K., Banthia, A. and Majumdar, D. (2007) Biomedical Evaluation of Polyvinyl Alcohol-Gelatin Esterified Hydrogel for Wound Dressing. Journal of Materials Science: Materials in Medicine, 18, 1889-1894. http://dx.doi.org/10.1007/s10856-007-3061-2

[32] Maria, T.M., De Carvalho, R.A., Sobral, P.J., Habitante, A.M.B. and Solorza-Feria, J. (2008) The Effect of the Degree of Hydrolysis of the PVA and the Plasticizer Concentration on the Color, Opacity, and Thermal and Mechanical Properties of Films Based on PVA and Gelatin Blends. Journal of Food Engineering, 87, 191-199. http://dx.doi.org/10.1016/j.jfoodeng.2007.11.026

[33] Mendieta-Taboada, O., Sobral, P.J.D.A., Carvalho, R.A. and Habitante, A.M.B. (2008) Thermomechanical Properties of Biodegradable Films Based on Blends of Gelatin and Poly (Vinyl Alcohol). Food Hydrocolloids, 22, 1485-1492. http://dx.doi.org/10.1016/j.foodhyd.2007.10.001

[34] Pal, K., Banthia, A. and Majumdar, D. (2006) Polyvinyl Alcohol—Gelatin Patches of Salicylic Acid: Preparation, Characterization and Drug Release Studies. Journal of Biomaterials Applications, 21, 75-91. http://dx.doi.org/10.1177/0885328206056312

[35] Bajpai, A. and Saini, R. (2005) Preparation and Characterization of Biocompatible Spongy Cryogels of Poly (Vinyl Alcohol)—Gelatin and Study of Water Sorption Behaviour. Polymer international, 54, 1233-1242. http://dx.doi.org/10.1002/pi.1813

[36] You, S.J., Ahn, W.S., Jang, H.S., Kang, M.I., Chun, H.J., Lim, Y.M., et al. (2007) Preparation and Characterization of Gelatin-Poly (Vinyl Alcohol) Hydrogels for Three-Dimensional Cell Culture. Journal of Industrial and Engineering Chemistry, 13, 116-120.

[37] Pawde, S. and Deshmukh, K. (2008) Characterization of Polyvinyl Alcohol/Gelatin Blend Hydrogel Films for Biomedical Applications. Journal of Applied Polymer Science, 109, 3431-3437. http://dx.doi.org/10.1002/app.28454

[38] Pal, K., Banthia, A.K. and Majumdar, D.K. (2007) Preparation and Characterization of Polyvinyl Alcohol-Gelatin Hydrogel Membranes for Biomedical Applications. Aaps Pharmscitech, 8, E142-E146. http://dx.doi.org/10.1208/pt080121

[39] Van der Linden, J.C., Waarsing, J.H. and Weinans, H. (2006) The Use of Micro-CT to Study Bone Architecture Dynamics Noninvasively. Drug Discovery Today: Technologies, 3, 213-219. http://dx.doi.org/10.1016/j.ddtec.2006.06.006

[40] Park, S.Y., Kim, K.H., Koo, K.T., Lee, K.W., Lee, Y.M., Chung, C.P., et al. (2011) The Evaluation of the Correlation between Histomorphometric Analysis and Micro-Computed Tomography Analysis in AdBMP-2 Induced Bone Regeneration in Rat Calvarial Defects. Journal of Periodontal \& Implant Science, 41, 218-226. http://dx.doi.org/10.5051/jpis.2011.41.5.218 


\section{Submit or recommend next manuscript to SCIRP and we will provide best service for you:}

Accepting pre-submission inquiries through Email, Facebook, LinkedIn, Twitter, etc.

A wide selection of journals (inclusive of 9 subjects, more than 200 journals)

Providing 24-hour high-quality service

User-friendly online submission system

Fair and swift peer-review system

Efficient typesetting and proofreading procedure

Display of the result of downloads and visits, as well as the number of cited articles

Maximum dissemination of your research work

Submit your manuscript at: http://papersubmission.scirp.org/ 